\title{
COVID-19 Expands Its Territories from Humans to Animals
}

\author{
George F. Gao ${ }^{1,2, \#}$; Liang Wang ${ }^{2}$
}

\section{THE GLOBAL STATUS OF COVID-19 PANDEMIC}

Since the discovery of a novel type of coronavirus named severe acute respiratory syndrome coronavirus 2 (SARS-CoV-2, also known as 2019-nCoV, or $\mathrm{HCoV}-19$ ) (1), the causative agent of coronavirus disease 2019 (COVID-19), COVID-19 has been spreading globally during a short period of time. As of August 17, COVID-19 had caused more than 206 million infections, of which more than 4 million had died worldwide (2), which is the worst pandemic caused by coronaviruses thus far. The pandemic of COVID-19 has not only posed a global threat to public health but has also thoroughly taxed medical systems and global economies. In response to this pandemic, unprecedented efforts have been made worldwide, such as the implementation of nonpharmacological interventions (NPIs) (3) and vaccine development (4). However, several types of variants of concern (VOC) have been found gradually during the circulation of SARS-CoV-2, as the combination effects of their intrinsic characteristics of error-prone replication process, host immune pressure and other environmental factors. Several studies have documented that these VOCs showed increased transmissibility and were more resistant to neutralization by convalescent and vaccine sera than other variants, posing a global threat to public health. Taken together, the arms race between SARS-CoV-2 evolution and human coping strategies will continue for some time to come.

\section{THE ORIGIN OF SARS-COV-2 REMAINS A MYSTERY}

For any causative agent of a pandemic, tracing the origin of the "prime criminal" is crucial for both understanding its evolution and preventing outbreaks in the future. To our knowledge, a bat-origin coronavirus RaTG13 had the most similar genome compared to SARS-CoV-2 at the whole genomic level
(96.2\%), although the amino acid similarity of the receptor binding domain (RBD) of the spike protein, which mediates the viral entry to human cell, was only $89.2 \%$. RaTG13 pseudovirus could transduce cells expressing human ACE2 with low efficiency (5). Subsequently, several coronavirus genomic sequences with high similarity to SARS-CoV-2 from bats have been found in different countries (6-8). Both pseudotyped SARS-CoV-2 virus and wild SARS-CoV-2 virus could infect cells expressing ACE2 from Rhinolophus macrotis (9). These results indicated that SARS-CoV-2 could have more likely originated from bats. However, it is well established that intermediate hosts are needed for bat-origin coronaviruses before they acquire sufficient mutations to have the ability to infect humans, such as dromedary camels for MERSCoV. Two groups reported that they found SARS$\mathrm{CoV}$-2-related coronaviruses in Malayan pangolins (Manis javanica) and their RBD region had a high similarity to that of SARS-CoV-2. Yet, the overall genomic similarity compared to SARS-CoV-2 were both low $(<93 \%)$, which suggested pangolins were unlikely to be the intermediate host for SARS-CoV-2. So far, the mysteries of the route through which SARS$\mathrm{CoV}$-2-related coronaviruses were transmitted from bats to humans and if bats were the original reservoir host still remain unsolved.

\section{THE SPILLOVER OF SARS-CoV-2 EVENTS AND THEIR POTENTIAL MOLECULAR MECHANISMS}

In addition to humans, natural infection of SARS$\mathrm{CoV}-2$ have been found in several other species of mammals via contact with COVID-19 patients, such as cats, dogs, lions and tigers in zoos, minks, and ferrets (10) (Figure 1A). Snow leopards, pumas, and gorilla also have been found to be infected with SARS-CoV-2 in nature (10) (Figure 1A). Among these spillover events, mink-related SARS-CoV-2 variants had the greatest impact, as the mink had also transmitted the SARS-CoV-2 variants back to humans and caused 

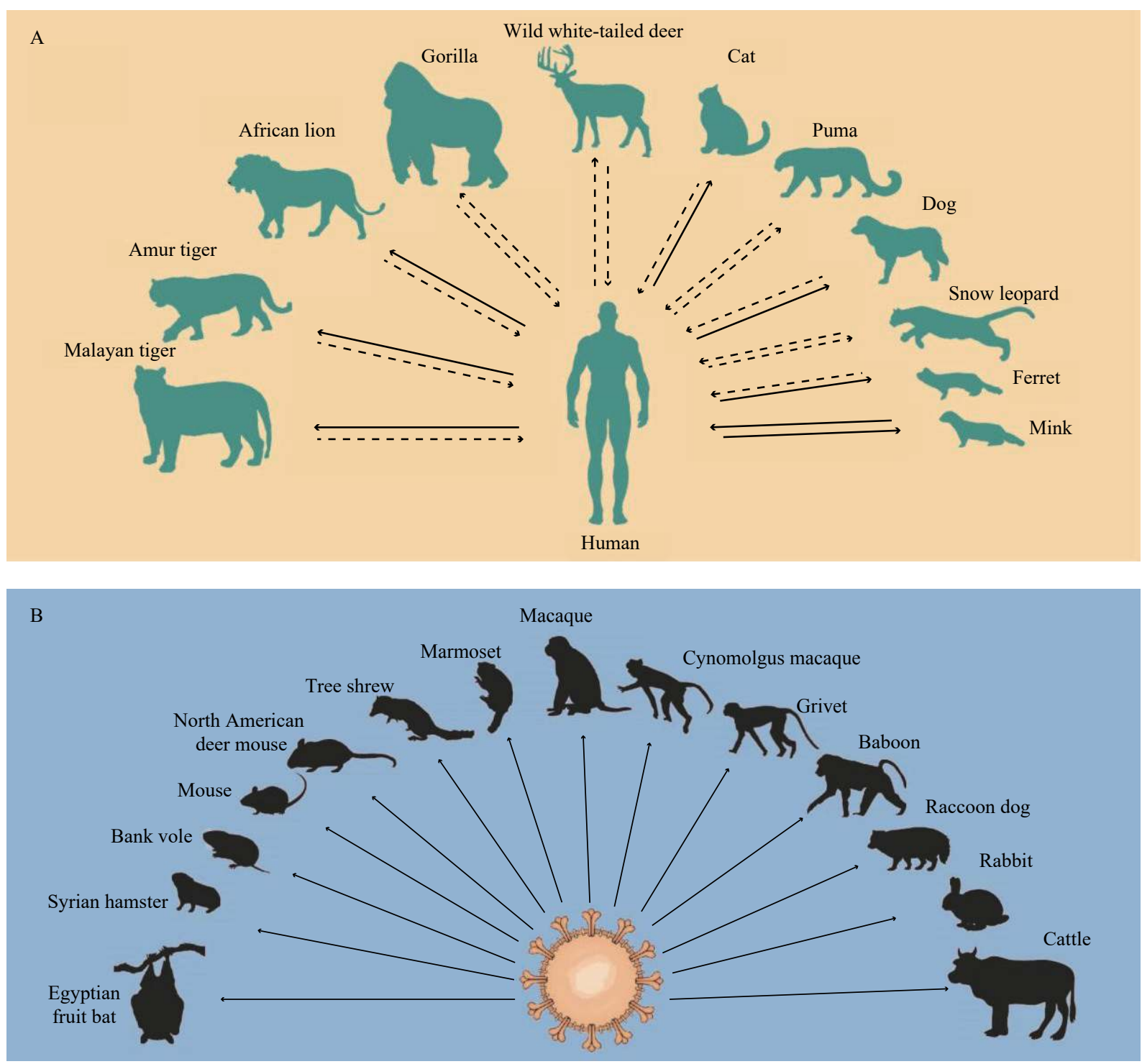

FIGURE 1. The currently known host range of SARS-CoV-2. (A) Species that have been reported to be infected by SARSCoV-2 in nature. (B) Species that have reportedly been infected by SARS-CoV-2 via infection experiments.

Note: A solid arrow indicates that there is clear evidence showing the cross-species transmission direction of SARS-CoV-2, while a dashed arrow indicates a possible cross-species transmission direction of SARS-CoV-2.

further community transmission (11). In order to prevent the mink-derived SARS-CoV-2 variants from continuously both circulating in the minks and spreading to humans, tens of millions of minks have been culled which also caused huge economic losses to mink-related industries. In addition to natural infection cases, several animals (e.g., rabbits, pigs, foxes, and civets etc.) have also been considered as possibly susceptible hosts of SARS-CoV-2 through infection experiments (10) (Figure 1B). Although most natural infections described above occurred in domesticated animals, far fewer cases were identified in the wild.

Recently, serosurveillance showed that antibodies against SARS-CoV-2 have been found in $40 \%$ of samples of wild white-tailed deer (Odocoileus virginianus) from USA in 2021 (12). However, antibodies were detected in only 1 and 3 samples from 2019 and 2020, respectively (12). Although it is still not known if SARS-CoV-2 in wild white-tailed deer was introduced by humans, the significant increase of positive rate of antibodies indicated that SARS-CoV-2 had been circulating in wild white-tailed deer. Due to the wide geographic distribution and large population (approximately 30 million) of wild white-tailed deer in North America, contact between human and wild white-tailed deer could be achieved by several activities such as wildlife rehabilitation, field research, practical 
conservation work and some wildlife-related tourism, supplemental feeding, captive cervid operations, and hunting (13). In this case, it increased the risk that SARS-CoV-2 from wildlife would spillback to humans.

Structural and functional studies identified several key sites from both SARS-CoV-2-RBD and ACE2. Mutations at these sites are closely related to the crossspecies transmission of SARS-CoV-2. On SARS-CoV2-RBD, residues 493, 498, and 501 were identified as key mutation hotpots that determine the host range. Mouse-adapted strains with N501Y mutation on RBD were isolated after 6 passages cultured in aged mice by wild type SARS-CoV-2 (14). Another 2 mutations, namely Q493K and Q498H were introduced to the S protein in mouse-adapted strains. Retrospective analysis showed that Q493K began to appear after 5 passages and, notably, Q498H dominated after only 1 passage. On ACE2, residues 41 and 42 were recognized as the critical sites for SARS-CoV-2 RBD binding. Sequence alignment showed that these two residues account for the major difference in the interaction network with SARS-CoV-2 RBD among different ACE2 orthologs (9). There are 4 combinations at these 2 sites (Y41-Q42, H41-Q42, Y41-E42, and H41-E42). Among them, Y41-Q42 combination showed the highest binding affinity to SARS-CoV-2 RBD; H41-Q42 showed much weaker; Y41-E42 was weaker than that of H41-Q42; and H41E42 showed the weakest binding to SARS-CoV-2 RBD. In addition, K31 and K353 have been reported as a hotspot providing substantial amount of energy in receptor binding, and the mutation from $\mathrm{K} 353$ to A353 may abolish the binding capacity of ACE2 to SARS-CoV-2-RBD.

\section{THE HOST EXPANSION OF SARS-COV-2 IS NOT OVER}

The host expansion of coronaviruses was well established (15). A previous study found some ongoing mink-adapted mutations such as Y453F, F486L, and N501T in the $S$ protein; for example, Y453F has been found to increase in hACE-2 affinity (16). Those results suggested that some ongoing mink-adapted mutations posed a huge threat to public health if they transmitted back to humans and even triggered further community transmission. Due to the fact that minks are bred in farms, large-scale slaughter of these minks can effectively prevent mink-derived SARS-CoV-2 variants from spreading and mutation accumulation in the mink population. However, similar measures could not be taken for wild animals. Together with the fact that adaptive mutations are needed when cross-species transmission happens and then circulate among populations of the new host, more efforts are needed to survey the genetic alterations and corresponding impact of transmissibility and infectivity in humans in these novel variants from wild white-tailed deer. Since SARS-CoV-2 is going wild, many other wild animals would also be infected with SARS-CoV-2 via direct or indirect contact with wild white-tailed deer or even infected patients. Several experimental studies have demonstrated several animals could be susceptible to SARS-CoV-2, such as Egyptian fruit bats (Rousettus aegyptiacus), marmosets (Callithrix jacchus), macaques (Macaca fascicularis and Macaca mulatta), bank voles (Myodes glareolus), and North American deer mice (Peromyscus maniculatus) (10). However, these are just the tip of the iceberg as the susceptibility of most terrestrial wild animals to SARS-CoV-2 has not been tested. In addition, the research on susceptibility of marine wildlife (especially marine mammals) to SARS$\mathrm{CoV}-2$ is still lacking. Due to frequent marine human activities (such as mariculture and marine fishing), the frequency of human contact with marine organisms is high. If some marine organisms are highly susceptible to SARS-CoV-2, there is a risk that SARS-CoV-2 could be transmitted from humans to marine organisms, and worse, SARS-CoV-2 then might spread in the marine ecosystem, which may lead to the generation of some novel SARS-CoV-2 variants with unknown threats to humans. Therefore, it is necessary to carry out large-scale SARS-CoV-2 screening for terrestrial and marine wildlife, especially those susceptible ones, in order to monitor the status of infection and mutation of SARS-CoV-2 in wild animals, so as to formulate further prevention and control strategies. It also provides more clues to the study of the origin and cross-species transmission of SARS-CoV-2.

Acknowledgement: Dr. Kefang Liu and Mr. Linjie Li for help in collecting the references and drawing figure.

Funding: Supported by the Strategic Priority Research Program of the Chinese Academy of Sciences (XDB29010202) and the intramural special grant for SARS-CoV-2 research from the Chinese Academy of Sciences.

doi: $10.46234 / \mathrm{ccdcw} 2021.210$

\# Corresponding author: George F. Gao, gaof@im.ac.cn. 
${ }^{1}$ Chinese Center for Disease Control and Prevention, Beijing, China; ${ }^{2}$ CAS Key Laboratory of Pathogen Microbiology and Immunology, Institute of Microbiology, Center for Influenza Research and Earlywarning, CAS-TWAS Center of Excellence for Emerging Infectious Diseases, Chinese Academy of Sciences, Beijing, China.

Submitted: August 24, 2021; Accepted: September 06, 2021

\section{REFERENCES}

1. Tan WJ, Zhao X, Ma XJ, Wang WL, Niu PH, Xu WB, et al. A novel coronavirus genome identified in a cluster of pneumonia cases Wuhan, China 2019-2020. China CDC Wkly 2020;2(4):61-2. http://dx.doi.org/10.46234/ccdcw2020.017.

2. World Health Organization. Coronavirus disease (COVID-19) weekly epidemiological update and weekly operational update. 2021. https:// www.who.int/emergencies/diseases/novel-coronavirus-2019/situationreports. [2021-08-05].

3. Li ZJ, Chen QL, Feng LZ, Rodewald L, Xia YY, Yu HL, et al. Active case finding with case management: the key to tackling the COVID-19 pandemic. Lancet 2020;396(10243):63 - 70. http://dx.doi.org/10.1016/ S0140-6736(20)31278-2.

4. Dai LP, Gao GF. Viral targets for vaccines against COVID-19. Nat Rev Immunol 2021;21(2):73 - 82. http://dx.doi.org/10.1038/s41577-02000480-0.

5. Liu KF, Pan XQ, Li LJ, Yu F, Zheng AQ, Du P, et al. Binding and molecular basis of the bat coronavirus RaTG13 virus to ACE2 in humans and other species. Cell 2021;184(13):3438 - 51.e10. http://dx. doi.org/10.1016/j.cell.2021.05.031.

6. Murakami S, Kitamura T, Suzuki J, Sato R, Aoi T, Fujii M, et al. Detection and characterization of bat Sarbecovirus phylogenetically related to SARS-CoV-2, Japan. Emerg Infect Dis 2020;26(12):30259. http://dx.doi.org/10.3201/eid2612.203386.

7. Hul V, Delaune D, Karlsson EA, Hassanin A, Tey PO, Baidaliuk A, et al. A novel SARS-CoV-2 related coronavirus in bats from Cambodia. bioRxiv, 2021. http://dx.doi.org/10.1101/2021.01.26.428212.

8. Zhou H, Ji JK, Chen X, Bi YH, Li J, Wang QH, et al. Identification of novel bat coronaviruses sheds light on the evolutionary origins of SARSCoV-2 and related viruses. Cell 2021;184(17):4380 - 91.e14. http://dx. doi.org/10.1016/J.CELL.2021.06.008.

9. Liu KF, Tan SG, Niu S, Wang J, Wu LL, Sun H, et al. Cross-species recognition of SARS-CoV-2 to bat ACE2. Proc Natl Acad Sci USA 2021;118(1):e2020216118. http://dx.doi.org/10.1073/PNAS.2020216 118

10. World Organization for Animal Health. Infection with SARS-CoV-2 in animals. 2021. https://www.oie.int/app/uploads/2021/05/en-factsheetsars-cov-2.pdf.[2021-08-05].

11. Munnink BBO, Sikkema RS, Nieuwenhuijse DF, Molenaar RJ, Munger E, Molenkamp R, et al. Transmission of SARS-CoV-2 on mink farms between humans and mink and back to humans. Science 2021;371(6525):172 - 7. http://dx.doi.org/10.1126/science.abe5901.

12. Chandler JC, Bevins SN, Ellis JW, Linder TJ, Tell RM, Jenkins-Moore $\mathrm{M}$, et al. SARS-CoV-2 exposure in wild white-tailed deer (Odocoileus virginianus). bioRxiv, 2021. http://dx.doi.org/10.1101/2021.07.29. 454326.

13. Delahay RJ, de la Fuente J, Smith GC, Sharun K, Snary EL, Girón LF, et al. Assessing the risks of SARS-CoV-2 in wildlife. One Health Outlook 2021;3:7. http://dx.doi.org/10.1186/s42522-021-00039-6.

14. Huang K, Zhang YF, Hui XF, Zhao Y, Gong WX, Wang T, et al. Q493K and Q498H substitutions in spike promote adaptation of SARS-CoV-2 in mice. EBioMedicine 2021;67:103381. http://dx. doi.org/10.1016/j.ebiom.2021.103381.

15. Wang L, Su S, Bi YH, Wong G, Gao GF. Bat-origin coronaviruses expand their host range to pigs. Trends Microbiol 2018;26(6):466 - 70. http://dx.doi.org/10.1016/j.tim.2018.03.001

16. Bayarri-Olmos R, Rosbjerg A, Johnsen LB, Helgstrand C, BakThomsen T, Garred P, et al. The SARS-CoV-2 Y453F mink variant displays a pronounced increase in ACE-2 affinity but does not challenge antibody neutralization. J Biol Chem 2021;296:100536. http://dx. doi.org/10.1016/j.jbc.2021.100536. 